\title{
'Tis Pity She's a Whore : la justice divine comme élément de l'esthétique baroque
}

Jean-Pierre Maquerlot

Marie-Thérèse Jones-Davies (éd.)

\section{(2) OpenEdition}

Journals

Édition électronique

URL : http://journals.openedition.org/shakespeare/1132

DOI : 10.4000/shakespeare.1132

ISSN : 2271-6424

Éditeur

Société Française Shakespeare

Édition imprimée

Date de publication : 1 novembre 1980

Pagination : 131-148

Référence électronique

Jean-Pierre Maquerlot, «'Tis Pity She's a Whore : la justice divine comme élément de l'esthétique baroque ", Actes des congrès de la Société française Shakespeare [En ligne], 2 | 1980, mis en ligne le 01 novembre 2007, consulté le 06 mai 2019. URL : http://journals.openedition.org/shakespeare/1132 DOI : 10.4000/shakespeare.1132 


\section{SOCIÉTÉ FRANC̣AISE SHAKESPEARE}

\section{ACTES DU CONGRĖS 1980}

DIRECTEUR DE LA PUBLICATION

M.T. Jones - Davies

JEAN TOUZOT Libraire - Editeur 38 , rue Saint-Sulpice 75278 PARIS CEDEX 061981 


\section{'TIS PITY SHE'S A WHORE : LA JUSTICE DIVINE COMME ELEMENT DE L'ESTHETIQUE BAROQUE}

Parler en 1980 de théâtre baroque autrement que d'une façon métaphorique ou mondaine, c'est-à-dire prétendre saisir à travers ce mot la spécificité d'un style, n'est plus faire preuve d'une témérité inexcusable ou d'une intention subversive. Grâce aux recherches menées autour du baroque depuis le début du siècle, la notion a perdu beaucoup de sa vertu provocatrice. Suite à la parution en 1915 des Principes Fondamentaux de l'histoire de l'art ${ }^{1}$ de Heinrich Wölfflin qui confèrent au baroque sa légitimité en tant que catégorie stylistique appliquée aux arts visuels, une abondante littérature s'est développé qui s'efforce de transposer cette catégorie aux arts du langage, au théâtre et à la musique. La procédure la plus "couramment suivie a consisté à adapter à ces formes d'art tout ou partie des concepts wölffliniens ${ }^{2}$, ce qui n'est pas sans poser de difficiles problèmes sur le plan théorique tant il est vrai qu'on ne peut impunément et innocement appliquer aux arts non visuels ou non exclusivement visuels comme le théâtre des concepts élaborés à partir de l'architecture, de la peinture et de la sculpture.

Il n'est pas dans mon propos de recenser et encore moins de «traiter» les problèmes complexes que pose un tel transfert, problèmes qui, pour avoir été souvent mal formulés ${ }^{3}$ et parfois ignorés, ont rendu la recherche baroquiste stérile, quand ils ne l'ont pas discréditée. Je ne peux davantage retracer ici l'histoire des avancées et des reculs de cette recherche, ce qui m'entraînerait trop loin du sujet annoncé pour cet exposé. Je me bornerai donc à constater que s'agissant du domaine qui nous est commun - la littérature et le théâtre de la Renaissance anglaise - le baroque n'a pas encore conquis son droit de cité dans le discours dominant de la critique et de l'histoire littéraire, sans doute parce qu'il n'a pas encore prouvé suffisamment son utilité. Trois raisons principales expliquent les résistances à la pénétration de cette catégorie dans le champ de notre discours critique.

1 - D'abord les anglicistes, tout comme les autres «littéraires», éprouvent une méfiance salutaire à l'égard d'une catégorie qui est un produit importé d'un domaine qui n'est pas le leur : celui des arts plastiques. Ils savent les 
difficiles problèmes que pose la «correspondance des arts», pour reprendre le titre d'un desouvrages les plus marquants d'Etienne Souriau ${ }^{4}$, et ils n'ignorent pas combien il est illusoire de parler de baroque en littérature ou au théâtre si l'on s'interdit, sous prétexte que le terrain est glissant, de se référer aux arts visuels. On ne peut en effet faire comme si le mot était d'emblée et de plein droit pertinent à la littérature et au théâtre. Dire qu'un poème ou qu'une pièce de théâtre est baroque est une proposition dont on doit bien mesurer le caractère insolite, même si elle n'est plus le fait de quelques contestataires à la mode. Il faut pouvoir se justifier et cela ne se peut que si l'on a préalablement mis au point une procédure de rapprochements analogiques qui, à la fois, respecte la spécifité de chaque art et détermine dans quelle mesure, à quels niveaux et sous quelles formes des correspondances sont possibles.

2 - La seconde raison des réticences tient à l'imprécision du mot dès lors qu'il est soustrait à ses déterminations plastiques et qu'il s'installe allègrement dans le parler quotidien, où il peut aussi bien qualifier une recette de cuisine, une manière de marquer des buts ou une prise de position politique; je renvoie ici au livre fort divertissant de Pierre Charpentrat, Le mirage baroque 5 . L'inflation d'un vocable s'accompagne toujours d'une dévaluation sémantique. Accommodé à toutes les sauces, le baroque est devenu le plus petit dénominateur commun pour tout ce qui, ici ou là, semble bizarre, extravagant, illogique, mouvementé, torturé, ostentatoire etc... On ne s'étonnera pas que face à cette banalisation du mot et aux risques de contamination par le vague que son utilisation déréglée dans le discours quotidien fait courir au discours «savant», nombreux soient ceux qui le tiennent pour nocif. Au demeurant d'excellentes analyses sur' la littérature et le théâtre anglais de notre période ont montré avec éclat qu'on peut faire l'économie du baroque et enrichir notablement notre connaissance de l'art littéraire et théâtral de l'époque ${ }^{6}$. Cette dernière observation me conduit à évoquer la troisième raison qui, cette fois, nous est plus particulière et explique l'insuccès du baroque.

3 - Le baroque est chez nous en concurrence avec d'autres catégories plus anciennes qui ont démontré leur valeur heuristique. Ce n'est pas en vain qu'on parle couramment de littérature et de théâtre «élisabéthains», «jaco- 
béens», «caroléens» ou de style «métaphysique». Les trois premières catégories restent des outils assez rudimentaires de périodisation historique mais elles sont chargées de connotations stylistiques et elles rendent compte d'une évolution réelle des formes et des contenus, même s'il n'échappe à personne que les étapes de cette évolution ne coincident pas parfaitement avec la succession des règnes. Les théâtres élisabéthain, jacobéen, caroléen apparaissent comme les sous-ensembles d'un ensemble plus vaste appelé théâtre de la Renaissance et, de ce point de vue, les catégories existantes ont l'avantage de nous faire saisir dialectiquement ce qui distingue Kyd de Webster et de Shirley et ce qui, au-delà des différences imputables aux individualités et à l'évolution des styles, les apparente à une tradition commune, celle du théâtre de la Renaissance, tradition qui, moyennant transformations, se maintient jusqu'en 1642. Quant à la notion de style métaphysique, elle dénote un phénomène littéraire qui reflète une modification dans la conception et la pratique de l'écriture (elle-même liée à des transformations d'ordre idéologique), mais elle connote un moment de l'évolution du style. Cette catégorie prioritairement appliquée à la poésie mais dont il faudrait continuer d'approfondir la pertinence à l'art du théâtre ${ }^{7}$ permet elle aussi une appréhension féconde d'un certain type de sensibilité, d'un mode de penser et d'écrire. En particulier elle donne à voir la manière dont un poète comme John Donne et un poètedramaturge comme Shakespeare à la fois recueillent et contestent, selon des modalités qui leur sont propres, l'héritage de rhétoriques antérieures comme le pétrarquisme, l'euphuisme, le spensérianisme, le style de Kyd et de Marlowe. On voit donc que les analystes de la littérature et du théâtre n'ont pas attendu le baroque et n'ont en général pas compté sur le baroque pour produire une représentation très affinée de la réalité qu'ils étudient.

A quoi bon dès lors recourir à cette notion d'un maniement si redoutable ? L'objet de mon intervention est de montrer sur un exemple théâtral précis, 'Tis Pity She's a Whore de Ford, pièce écrite à une date inconnue entre 1626 et 1633 , que la notion de baroque, sous certaines conditions d'emploi, peut s'avérer utile, être, pour ainsi dire, le révélateur de traits thématiques et formels qui, autrement, risqueraient d'échapper à l'observation. Qu'attendre au fond 
d'une expérience comme celle-ci sinon que le baroque fonctionne comme un dispositif optique qui oriente le regard vers certains aspects de la réalité que l'utilisation répétée d'autres catégories - d'autres lunettes - avaient quelque peu occultés ? Aussi me pardonnera-t-on d'avoir légèrement tiré à moi le sujet de ce colloque en faisant du thème de la justice et en particulier de la justice divine le prétexte de ma démonstration. Nous verrons que ce thème de la justice divine, à cause de son statut particulier dans notre pièce, signale la présence du baroque. Mais d'abord qu'est-ce que le baroque ? Question incontournable à laquelle il faut bien répondre au risque de retarder l'étude de 'Tis Pity. L'apport de. Jean Rousset à la recherche baroquiste me paraît encore à l'heure actuelle l'acquis le plus précieux dont nous disposons. On connaît les thèses développées dans le livre le plus connu de Rousset : La littérature de l'âge baroque en France, Circé et le Paon, paru en $1954^{8}$. L'auteur s'emploie à découvrir dans des formes d'art différentes (littérature, théâtre, ballet de cour, architecture, peinture, sculpture) des convergences thématiques et formelles d'où il ressort que le baroque est un art du changement, de l'inconstance, du spectacle etc... Circé et le Paon sont les figures symboliques qui incarnent respectivement la métamorphose et l'ostentation, les deux pôles de la sensibilité et de l'esthétique baroques. L'objection qu'inspire la démarche de Rousset est que l'inventaire ainsi constitué forme un ensemble de critères trop imprécis pour garantir que dans des proportions variables on ne puisse les retrouver dans des œuvres chronologiquement fort éloignées de l'époque dite baroque.Il n'est que de songer, entre mille exemples, à l'église de la Sainte Famille de Gaudi à Barcelone, aux grilles du métro par Guimard, au théâtre de Claudel ou d'Audiberti. S'en tenir à une définition «quantitative» du baroque fondée sur un simple constat de récurrence de formes et de thèmes, c'est évidemment s'exposer à rencontrer le baroque à tout moment et en tout lieu, n'avoir de ce style qu'une idée appauvrie qui, sous couvert de similitudes réelles mais superficielles, masque la spécificité du baroque historique, celui qui naît en Europe dans le sillage de la Renaissance ${ }^{9}$.

Dans un court texte beaucoup moins connu, intitulé «Peut-on définir le baroque ? ${ }^{10}$, J. Rousset approfondit 
l'analyse et esquisse une définition du baroque qui cerne davantage la spécificité historique du phénomène. Il considère quatre réalisations baroques : la fontaine des Quatre Fleuves du Bernin, l'église Saint-Yves de la Sapience de Borromini, un poème de Drelincourt et un passage de l'oraison funèbre d'Anne de Gonzague par Bossuet; et Rousset observe que ces œuvres, en dépit des différences inscrites dans le matériau lui-même, exhibent un mode d'organisation analogue. Faute de pouvoir par manque de temps rapporter pour chacun des cas étudiés les analyses de Rousset, je me contenterai de citer le passage le plus éclairant; il concerne Saint-Yves mais, dans ses grandes lignes, reproduit - mutatis mutandis - les conclusions tirées de l'étude de la fontaine, du poème et de l'extrait de l'oraison funèbre : La zone inférieure, perçue d'emblée, apparaît mouvementée et fractionnée, le plan hexagonal semble voler en éclats, l'impression première est celle de la fragmentation et de la division, de l'incertitude des équilibres, d'une géométrie qui se désarticule; mais si le regard s'élève vers la coupole, il voit le cercle rompu et convulsé se transformer et se résorber dans le cercle pur et calme du lanternon qui symbolise l'éternité et l'unité divines; on passe de l'instable au stable et du multiple à l'un, et c'est ce passage, cette transmutation inscrite dans les formes architecturales, qui donne à l'ouvrage sa signification; nous sommes appelés à vivre, sur un parcours continu, une métamorphose qui mène du cercle rompu au cercle parfait, de la multiplicité du sensible à l'unité du sacré; l'un est saisi dynamiquement à travers le multiple.

Plus loin, suite à l'étude stylistique du poème de Drelincourt et du passage de Bossuet, Rousset ajoute :

Ici encore, on passe de la multiplicité et de l'instabilité du monde - et de la phrase - à la simplicité de la permanence ... Mais c'est le même passage, le même mouvement qui se fractionne et se dilate, suivi d'un violent renversement qui découvre un point fixe. ${ }^{11}$

Cette analyse appelle deux remarques : 
1 - La définition proposée maintenant n'est plus quantitative (recenser les traits du baroque) ni taxinomique (les classer sous les deux rubiques de la métamorphose et de l'ostentation) mais fonctionnelle et structurale. Elle met en évidence le principe qui régit l'organisation du matériau plastique et linguistique.

2 - Ce principe est dialectique. L'œuvre matérialise l'opposition entre deux plans hiérarchisés qui renvoient à deux isotopies distinctes : au niveau inférieur, le multiple, l'éclaté, le disparate, le mouvant, le sensible; au niveau supérieur, l'un, le stable, le spirituel. Cest la résolution, ou mieux, le dépassement des contradictions inhérentes au premier niveau qui assure le passage au niveau supérieur. Rousset dit du cercle «rompu et convulsé» qu'il se «résorbe» dans le cercle pur et calme.

Voici en quelques mots la définition ou, si l'on veut, le modèle théorique auquel il va s'agir de confronter la pièce de Ford considérée sous l'angle de la justice.

Le principe de base qui structure la pièce envisagée de ce point de vue me paraît être l'opposition constamment rappelée entre deux ordres de réalités : une réalité qui est l'univers du drame avec ses tensions, ses conflits, ses explosions de violence, ses méprises et ses accalmies éphémères et une réalité abstraite, invisible, inaccessible, présente à la conscience de certains personnages, ignorée par d'autres mais fortement ressentie par le spectateur de la pièce, je veux parler de la présence de Dieu à l'arrière-plan du drame. Cette présence se manifeste au niveau du discours par la forte récurrence de mots comme «God» et «heaven». Dans sa préface à 'Tis Pity. N.W. Bawcutt dénombre plus de trente occurences du mot «heaven» pour s'étonner que ces répétitions n'aient pas été mieux perçues par les critiques ${ }^{12}$. La présence de Dieu se manifeste aussi dans des retournements de situation comme le repentir d'Annabella, des coïncidences fortuites comme l'arrivée opportune du frêre Bonaventura à qui Annabella confie une lettre à l'intention de Giovanni. Il n'est pas douteux que les multiples allusions à Dieu qui préparent et accompagnent ces événements visent à nous les faire interpréter comme les signes tangibles de l'intervention divine, ce dont ne doute aucun des personnages impliqués dans ces événements. Or cette opposition très générale entre l'ici-bas et l'au-delà qui conditionne largement la conduite de l'action, le comportement des person- 
nages, l'investissement thématique et jusqu'à l'organisation même de certains énoncés ${ }^{13}$, trouve son expression la plus claire dans la manière dont la pièce traite de la justice.

Le thème figure sous deux aspects contradictoires : la justice humaine et la justice divine, celle administrée par les hommes et celle administrée par la Providence. Ces deux types de justice s'opposent comme les termes de la dialectique baroque. A l'étage inférieur, la justice humaine offre le triste spectacle de sa faillite et de sa malfaisance tandis que conjointement se trouve reconnue et exaltée l'existence d'une justice supérieure, celle du souverain Juge. Ainsi l'on assiste tout au long de la pièce à la mise en cuvre méthodique d'un processus de dévalorisation de la justice humaine et du processus inverse de glorification de la justice divine. Quels sont les aspects majeurs de cette double stratégie qui épouse le schéma du baroque?

Tout d'abord il y a la négation de la justice institutionnelle. Le seul personnage qui aurait qualité pour rendre la justice, c'est-à-dire parler et agir au nom de Dieu et de la société, est le Cardinal, légat du Pape; or celui-ci est totalement disqualifié par la protection scandaleuse qu'il accorde à Grimaldi, assassin par erreur de Bergetto, mais assassin tout de même. Ce déni de justice est ressenti comme tel par Donado et Florio et l'on remarquera, en contrepoint, l'affirmation que la seule vraie justice n'est plus de ce monde :

Donado : Is this a Churchman's voice? Dwell's justice here?

Florio : Justice is fled to Heaven and comes no nearer. (III,ix,61-62)

Le spectateur anglais contemporain avait évidemment toute raison de reconnaître dans la justice papiste un modèle d'iniquité. Le deuxième aspect de ce parti pris dévalorisant consiste à substituer à la justice selon la loi cette justice élémentaire primitive que Bacon appelait «sauvage» (wild justice» qui est la vengeance privée, acte impie aux regards de la loi civile et religieuse ${ }^{14}$.

Dans la Tragédie Espagnole, Hieronimo, Maréchal d'Espagne, fait partie de l'«establishment» judiciaire; son premier mouvement est de demander justice par les voies légales et c'est après l'échec de sa démarche qu'il s'en remet à la vengeance. Hamlet sait au départ qu'il ne peut compter que sur lui-même puisque l'autorité détentrice du pouvoir 
judiciaire est ici représentée par Claudius, l'usurpateur du trône et l'assassin du roi légitime. Ces personnages sont donc acculés à la vengeance par des circonstances qu'ils ne maîtrisent pas et ils ont pour agir de puissantes raisons : la mort d'un fils et la mort d'un père. A l'appel douloureux des pauvres âmes errantes qui ne peuvent trouver le repos, une seule réponse : la vengeance; de sorte que si l'acte de vengeance met les vengeurs au ban de la société civile et de la communauté chrétienne, il n'a pas sur eux d'effets humainement dégradants. Se venger, c'est le moyen, le seul, de satisfaire dans les tourments ou la jubilation, mais toujours avec la certitude de se perdre, un irrésistible besoin de justice.

Dans 'Tis Pity il en va autrement. Les cinq personnages en quête de vengeance n'ont aucune des raisons péremptoires qui poussent les héros tragiques à s'ériger en justiciers de leur propre cause. Grimaldi veut faire payer à Soranzo une blessure d'amour-propre (il a eu le dessous dans une altercation avec Vasques, le serviteur de Soranzo) et, par là-même, neutraliser un rival auprès d'Annabella. Hippolita veut faire payer à Soranzo, son ancien amant, l'humiliation qu'il lui inflige en lui préférant Annabella, au mépris de ses anciennes promesses. Richardetto est le mari de cette femme adultère; de retour à Parme après qu'on l'ait cru mort, le voici qui complote la mort de son rival. Soranzo complote la mort d'Annabella et de Giovanni quand il découvre les relations incestueuses du frère et de la sœur. Giovanni tue Annabella et Soranzo car il ne supporte pas de voir sa sœur repentante possédée par un autre que lui. Or toutes ces vengeances, sauf la dernière, échouent complètement ou partiellement. Grimaldi tue l'innocent et niais Bergetto à la place de Soranzo; Hippolita est prise à son propre piège grâce à Vasques qu'elle croyait avoir soudoyé et elle meurt du poison qu'elle destinait à son amant infidèle. Ces deux épisodes conduisent Richardetto à renoncer à sa vengeance. Soranzo voit sa vengeance lui échapper à moitié puisque Giovanni tue Annabella avant qu'il n'ait eu le temps de s'en charger. Seul Giovanni mène à bien sa vengeance : il tue Annabella et Soranzo avant de tomber lui-même sous le poignard de Vasques et des tueurs à gages. Mais s'agit-il dans le cas de Giovanni d'une véritable vengeance ? Bien que le motif officiel du meurtre d'Annabella soit le repentir de cette dernière en violation du serment de 
l'Acte I, Scène ii, tout porte à croire que la vengeance dans laquelle se drape Giovanni est en réalité une pose, un alibi, une justification a posteriori qui dissimule autre chose. L'insistance avec laquelle le jeune homme tente de se faire passer pour un vengeur épris de justice est suspecte. La répétition à trois reprises des mots «revenge» et «vengeance» au cours des deux dernières scènes de l'Acte $V$ camoufle en fait, l'irrépressible et indicible désir de posséder au-delà de la mort et pour l'éternité la femme aimée. J'en veux pour preuve les ultimes paroles de Giovanni mourant :

Wher'er I go, let me enjoy this grace,

Freely to view my Annabella's face (V.vi,107-108)

La triomphale apparition de Giovanni, héros de l'amour incestueux mais vengeur douteux, arborant à la pointe de sa dague le cœur de sa sœur, marque l'accomplissement symbolique de ce désir de possession absolue 15 .

Ainsi le motif de la vengeance qui occupe une place centrale et éminente dans la «revenge play» traditionnelle est ici banalisé, balkanisé et perverti. Il est rare et relativement nouveau de voir la vengeance traitée avec autant de dérision. On ne peut apprécier, à cet égard, l'originalité de Ford si l'on ne voit pas que cette dénonciation de la vengeance s'insère dans une stratégie qui vise à détruire le mythe de la justice humaine aussi bien sous sa forme légale que sauvage.

Conformément au schéma du baroque, la négation de la justice humaine a pour corollaire l'affirmation de la justice divine qui, selon le Deutéronome (XXII, 35) et l'Epitre de Saint Paul aux Romains (XII, 19), n'est autre que «la vengeance et la récompence de Dieu» 16 .

Comment se présente la justice divine ? Nous savons qu'aucun personnage ne peut officiellement l'incarner; la justice divine est transcendante, mais elle ne s'exerce pas par délégation et elle ne se manifeste pas non plus sous la forme d'un événement surnaturel qui viendrait attester, comme au cinquième acte du Dom Juan de Molière, l'intervention directe de la Providence. La conception de la justice qui sous-tend la pièce est anti-humaniste et anti-théocratique; elle diffère sur ce point de la doctrine de Saint Paul et de la pensée chrétienne en général ${ }^{17}$. Et pourtant la justice divine triomphe au dénouement. Une fois bouclé le cercle des vengeances individuelles, Donado s'écrie face aux cada- 
vres qui jonchent la scène :

Strange miracle of justice! (V.vi, 109)

Dans cet effrayant dénouement qui châtie les coupables et n'épargne pas complètement l'innocence, Donado voit la main de Dieu. C'est que la vraie, la seule justice, celle voulue par Dieu, ne se matérialise pas sous la forme d'une vengeance équitable qui viendrait compenser l'iniquité des vengeances privées; la vengeance de Dieu apparait ici comme le point d'aboutissement, la résultante des vengeances humaines; c'est à travers elles qu'elles opère; c'est de leur inefficacité et de leur faillibilité conjuguées qu'elle tire sa propre efficacité et sa propre équité. Voici ce qui précisément la rend incompréhensible et miraculeuse. L'exclamation de Donado marque l'instant décisif où les survivants du drame ont la révélation de ce qui s'est passé; tout au long de la pièce Dieu a été le véritable ordonnateur des péripéties, laissant à chaque personnage la liberté d'accomplir son destin conformément aux voies impénétrables de la Providence. Le spectateur, quant à lui, avait été préparé à cet instant de révélation grâce à un épisode significatif; je veux parler de la scène ii de l'Acte IV où l'on voit Richardetto constater l'échec de son entreprise et renoncer à sa vengeance. L'assassinat pàr erreur de Bergetto, la vengeance ratée d'Hippolita et son effet boomerang, le déni de justice commis par le Cardinal - soit deux méprises et une injustice - ont appris à Richardetto la vanité et la malfaisance de la justice humaine sous ses deux aspects. C'est pourquoi il renonce et son renoncement se double d'un acte de foi, un pari sur la justice divine que lui dictent à la fois sa raison et son cœur :

My wretched wife, more wretched in her shame Than in her wrongs to me, hath paid too soon The forfeit of her modesty and life;

And I am sure, my niece, though vengeance hover, Keeping aloof yet from Soranzo's fall, Yet he will fall, and sink with his own weight. I need not now - my heart persuades me so To further his confusion; there is One

Above begins to work ... (IV .ii, 1-9)

Quelques vers plus bas, Richardetto conseille à Philotis, sa nièce, de quitter l'agitation du monde pour la paix du couvent. L'empressement que met la jeune fille à se faire 
nonne montre qu'elle aussi est touchée par la grâce. Cette courte scène illustre à merveille la façon dont Dieu «travaille» pour reprendre l'expression imagée que Ford prête à son personnage. Deux méprises funestes et une iniquité ont pour effet d'arracher Richardetto à l'engrenage destructeur des vengeances et Philotis aux tentations du monde. Deux âmes sont sur la voie du salut. Parallèlement ces échecs de la justice humaine contribuent, au plan de l'action, à assurer le succès de la justice divine. En effet, le sursis providentellement accordé à Soranzo rend possible la scène suivante où l'on voit Annabella, touchée par le repentir, implorer à genoux le pardon de son mari - troisième sauvetage spirituel ? - tandis que lui, dissimule son désir de vengeance sous les apparences trompeuses de la clémence. Ce trait machiavélique inspiré par Vasques, joint au cynisme donjuanesque que le personnage a manifesté envers Hippolita à l'Acte II ${ }^{18}$, achève de rendre Soranzo haïssable et justifie son châtiment au cinquième acte. De même, le repentir d'Annabella commencé à l'Acte III pendant la scène de contrition, son acquiescement au projet de mariage qu'on lui impose, le sentiment qu'elle a de son indignité en tant qu'épouse, sa tentative pour amener son frère au repentir, tout ceci va dans le sens du rachat et témoigne de la miséricorde divine mais, en même temps, tout ceci rend inévitable l'expiation sanglante de la faute, c'est-à-dire l'avènement de la justice. L'âme peut-être sauvée mais le corps souillé doit périr et il périt de la main de Giovanni par qui il a péché. Ainsi le partenaire incestueux se fait, à son insu, l'instrument de la justice divine. Le Giovanni du début, en lutte contre lui-même, en proie au besoin de justifier sa déviance à coups d'arguments philosophiques, fait place à un personnage endurci, sûr de lui, orgueilleux, résolu à aller jusqu'au bout de son désir de possession, inaccessible à la pitié et au compromis, comme il sied à l'exécuteur des Hautes Oeuvres qu'il est, sans le savoir, devenu.

Mais ce que Giovanni réalise une fois constitue la raison d'être d'un autre personnage dont on n'a pas parlé : Vasques. Vasques jouit dans la pièce d'un statut particulier; il est le seul vengeur qui 11'agit pas pour son compte mais pour un autre, en l'occurrence Soranzo, son maître, qu'il sert avec une intelligence infaillible et un dévouement sans bornes. Il y a chez le personnage un désintéressement, une 
abnégation, un zèle inconditionnel à servir qui étonnent et ne cadrent pas tout à fait avec l'image-type du «villain» jacobéen qui nourrit sa rancour et caresse une ambition personnelle. Vasques ne souffre de rien, n'attend rien, n'espère rien.Il est psychologiquement vide, investi en totalité dans chacun de ses actes. A la fin de la pièce il parle de son devoir de fidélité à la mémoire du père de Soranzo, son ancien bienfaiteur, mais si ses allégations nous éclairent sur les motivations du personnage, elles n'expliquent pas la place primordiale et la fonction éminente qui lui sont dévolues par le dramaturge dans l'organisation des vengeances. L'hypothèse que je propose est que cet Espagnol venu d'ailleurs, étranger parmi les Italiens de Parme bien que mêlé à leurs intrigues, est en réalité l'agent dépêché par Dieu sur les lieux du drame. C'est lui qui fait avorter la vengeance d'Hippolita et tous les personnages, y compris la mourante, reconnaissent dans ce coup de théâtre le signe de la justice divine :

$\begin{array}{ll}\text { Omnes } & \text { : Wonderful justice! } \\ \text { Richardetto } & : \text { Heaven, thou art righteous. } \\ \text { Hippolita } & : \text { O, 'tis true; (IV. }, 87-89)\end{array}$

C'est Vasques qui conseille à Soranzo de feindre la pitié face à Annabella; c'est encore lui qui obtient de Putana le nom du père de l'enfant que porte sa femme; après quoi il ordonne à ses hommes de main d'arracher les yeux de la vieille entremetteuse. Une fois connue de Soranzo l'identité des deux coupables, il ne lui reste plus qu'à tendre le piège, sous la direction de Vasques. Grâce aux machinations de Vasques se met en place le dispositif qui provoque la catastrophe. A partir de l'Acte IV il est au centre de l'action et - coïncidence significative - c'est au moment où Richardetto renonce à agir que Vasques prend le relais. La carrière des deux personnages suit une courbe inverse. Tous les deux participent activement à l'organisation des vengeances et tous les deux sont au dénouement pratiquement absous de leur faute. Vasques est certes banni de la ville mais le bannissement n'est guère pour lui un châtiment car, Soranzo mort, rien ne le retient plus à Parme. Une fois sa mission accomplie, l'étranger s'en va. Quant à Richardetto, sa responsabilité dans le meurtre de Bergetto est totalement oubliée. Quelle étrange immunité au regard des critères de la simple justice humaine ! Comment l'expliquer ? Richardetto est 
épargné pour avoir compris assez tôt la volonté de Dieu et s'être démis de sa fonction de vengeur; Vasques est épargné pour avoir réalisé, à son insu, les plans de la Providence. Dans un monde bouleversé où l'Eglise et l'Etat n'assurent plus la médiation nécessaire entre Dieu et l'individu, il faut bien que certains hommes de la trempe des Vasques soient les fléaux de Dieu, les instruments involontaires et aveugles de sa colère, les agents impitoyables de sa justice.

Un proverbe portugais dit : «Dieu écrit droit avec des lignes courbes» 19 . On est tenté de dire : l'artiste baroque aussi. L'artiste baroque ne croit pas que la ligne droite soit le plus court chemin de l'homme à Dieu, du relatif à l'abso$\mathrm{lu}$, du contingent au nécessaire, du multiple à l'un. L'itinéraire baroque nous entraîne de courbes en contre-courbes, de contorsions en convulsions jusqu'à ce point ultime où s'annullent les négations et où tout se résorbe dans l'affirmation d'une positivité supérieure. On reconnaîtra dans ce schéma dynamique la trajectoire suivie par la justice dans 'Tis Pity. Est-ce à dire pour autant que la pièce est baroque? Il faudrait pour l'affirmer étudier l'œuvre sous plus d'un aspect et je ne suis pas sûr que le but de la recherche dans ce domaine soit de décerner des étiquettes. On constatera cependant que l'hypothèse de départ s'est révélée opératoire et qu'elle a enrichi notre compréhension d'un aspect non négligeable de l'œuvre.

Mais je voudrais introduire pour terminer une réserve importante. Si le dénouement de 'Tis Pity consacre le triomphe de la justice divine, la pièce ne se termine pas, comme on pourrait s'y attendre en bonne logique baroque, sur la reconnaissance définitive de la toute-puissance de Dieu. Après la brève illumination de Donado semblable à un moment d'apothéose (Strange miracle of justice), vient la retombée dans la confusion et l'imperfection de l'ordre humain personnifié par le Cardinal. C'est lui qui prend en main la situation pendant les quelques minutes qui nous séparent de la fin du spectacle. Il commence par supposer, à tort, que Vasques veut assassiner tout le monde :

Raise up the city; we shall be murdered all ! (V.vi, 110)

Il se méprend sur le rôle joué par Putana et lui attribue la responsabilité principale de ce qui s'est passé; de sorte que la sentence de mort par le feu paraît disproportionnée par 
rapport au délit; cela n'empêche pas Donado lui-même qui, il y a un instant, témoignait encore de l'avènement miraculeux de la justice divine, d'applaudir servilement à ce jugement fondé sur un malentendu. Le Cardinal confisque pour le compte de la papauté les biens de ceux qui sont morts, ce qui ajoute une touche sordide au tableau. Enfin et surtout, dans le distique qui conclut la pièce et en tire la morale, il qualifie Annabella de «whore» usant d'un mot qu'on ne trouve employé que par Soranzo dans un accès de fureur démente (IV. iii). Or le Cardinal n'a pas cette excuse; de sang-froid il demande sur le ton de l'interrogation pontifiante :

Of one so young, so rich in nature's store Who could not say, 'tis pity she's a whore ? (V. vi, 159-160)

Cet hommage funèbre qui jette l'opprobre sur le personnage témoigne de la part de celui qui le prononce non seulement d'une incompréhension profonde du personnage d'Annabella et du drame qui vient de se jouer mais aussi porte l'indice d'une médiocrité d'âme et d'esprit qui contraste avec la noblesse des deux personnages tragiques. Or ce sont ces mots de la fin qui donnent à la pièce son titre. On peut penser que Ford satisfaisait ainsi à une convention théâtrale qui, de surcroit, lui fournissait un titre spécialement «accrocheur»; mais on ne peut croire que ce titre contre lequel toute la pièce s'inscrit en faux exprime le jugement du dramaturge. J'y vois pour ma part une provocation verbale en forme d'antiphrase dont l'auteur nous invite après coup à mesurer toute la charge ironique. Tout se passe comme si Ford, et par le titre et par la retombée qui succède à la culmination baroque, souhaitait faire prendre conscience à son public que dans une société condamnée comme celle de Parme, l'acquiescement à la justice divine ne peut être qu'éphémère. Le triomphalisme baroque en souffre mais la vérité y trouve son compte. 


\section{NOTES}

1. Le titre original : Kunstgeschichtliche Grundbegriffe. Le livre fut traduit en français en 1933 par Claire et Marcel Raymond.

2. H. Wölfflin oppose Renaissance et Baroque à travers cinq couples antithétiques :

Représentation linéaire/Représentation picturale

Représentation par plans distincts/Représentation en profondeur

Forme close/Forme ouverte

Vision multiple/Vision globale

Clarté absolue/Obscuritéi relative.

Parmi les auteurs qui ont le plus œuvré en faveur d'une transposition des concepts wölffliniens à la littérature et au théâtre, citons au moins les noms de Wylie Sypher : Four Stages of Renaissance Style, New York, 1955 et Marcel Raymond : Baroque et renaissance poétique, Paris, 1955. L'auteur conduit une réflexion lucide sur les difficultés du transfert qui tempère utilement les enthousiasmes un peu moins rigoureux de W.Sypher.

3. Voir le livre de René Wellek : Concepts of Criticism, Yale, 1963 On y trouve quelques exemples d'analogies douteuses entre les arts plastiques et la littérature, contre lesquelles R. Wellek met en garde le chercheur.

4. Etienne Souriau : La correspondance des arts, Paris, 1947 (2ème ed. 1969)

5. Pierre Charpentrat : Le mirage baroque, Paris, 1967.

6. A cet égard la démonstration la plus probante nous est fournie par le grand livre de R. Ellrodt : Les poètes métaphysiques anglais, Paris, 1960 ( 2ème ed. 1972). La réflexion introductive sur les problèmes théoriques et méthodologiques que soulève l'utilisation de baroque est toujours d'une très grande actualité.

7. Sir Herbert Grierson et T.S. Eliot ont orienté la critique dans cette direction. On pourra se reporter à l'ouvrage de Patrick Cruttwell : The Shakespearean Moment, New York, 1960.

8. Le livre de J. Rousset a fait l'objet de cinq réimpressions entre 1954 et 1965 . Après un certain reflux des études baroquistes qui donne à penser que les Journées de Montauban ont vécu leurs plus belles heures pendant la décennie 1965-75, on assiste à un regain d'intérêt à l'égard du baroque et ce sont les historiens qui mènent le mouvement. 1978 voit la parution en format livre de poche de la thèse de Michel Vovelle : Pitié baroque et déchristianisation en Provence au 18 e Siècle. Ce travail soutenu en 1971 fut publié une première fois en 1973. Cette année est réédité l'ouvrage devenu classique de Victor-Louis Tapié : Baroque et clacissisme. La dernière réimpression de ce livre écrit en 1957 datait de 1972. Le baroque n'a pas fini de faire parler de lui.

9. Le champion de la conception d'un baroque transhistorique est Eugenio d'Ors : Du baroque, Paris, 1935. Sa thèse est que baroque et clacissisme sont deux modes de vision antagonistes et intemporels parce que inhérents aux structures - évidemment éternelles - de l'esprit humain. L'«éon» du baroque s'est incarné au cours des âges en 22 espèces successives depuis le Barocchus pristinus de l'homme des cavernes jusqu'au Barocchus posteabellicus des années 
1920. Sousjacent à cette théorie, on trouve le dualisme nietzschéen apollinien/ dyonisiaque.

10. Voir les Actes des Journées Internationales d'Etudes du Baroque de Montauban, Toulouse, 1965, pp. 19-23. La communication de Jean Rousset est reproduite légèrement modifiée sous le titre «Saint-Yves et les poètes»dans L'intérieur et l'extérieur, Paris, 1968.

\section{Voir Actes, pp. 21-23.}

12. 'Tis Pity She's a Whore, ed. by N.W. Bawcutt, Regents Renaissance Drama Series, Londres, 1966. Voir l'introduction, p. XVI. Nos références sont tirées de cette édition.

13. L'enoncé qui reproduit le mieux la démarche baroque est fourni par le distique héroi que qui conclut la tirade de Richardetto :

All human worldly courses are uneven;

No life is blessed but the way to Heaven (IV.ii, 20-21)

On y remarque l'opposition et le passage du' multiple à l'un, de la terre au ciel des embûches d'ici-bas à l'unique voie, celle qui mène à Dieu, le tout exprimé à travers une double négation qui, par le renversement de «but» s'ouvre sur l'affirmation finale.

14. L'essai no 4 «Of revenge» s'ouvre sur ce jugement non équivoque : «Revenge is a kinde of Wilde Justice, which the more Man's Nature runs to, the more ought Law to weed it out. For as for the first Wrong, it doth but offend the Law; but the Revenge of that Wrong putteth the Law out of Office.» F. Bacon : Essais, Aubier (trad. par M. Castelain), Paris, 1948, p. 18.

15. Cette scène finale où l'on voit le héros «gilt in the blood/ Of a fair sister and a hapless father»(68-69)tenir en guise de sceptre la dague surmontée du cœur d'Annabella est l'apothéose baroque de la carrière amoureuse de Giovanni. Vainqueur' du temps, soustrait aux remords de la conscience, aux blames de la religion et de la société, Giovanni vit dans l'exaltation le moment de son triomphe; ce n'est pas en vain que son discours abonde en termes qui dénotent la victoire et la royauté («triumph», «glory», «monarch», «royally»...)

16. Deuteronomy (XXII, 35) : «To me belongeth vengeance, and recompence». The Epistle of Paul the Apostle to the Romans (XII, 19) : «Dearly beloved, avenge not yourselves, but rather give place unto wrath : for it is written, Vengeance in mine; I will repay, saith the Lord.»

17. Romans (XIII, 4) : "For he is the minister of God to thee for good. But if thou do that which is evil, be afraid; for he beareth not the sword in vain : for he is the minister of God, a revenger to execute wrath upon him that doeth evil.»

18. Voir 'Tis Pity, II .rí, 94-99

19. Deus escreve direito por linhas tortas. Le proverbe figure en exergue du Soulier de Satin de Paul Claudel. 